\title{
Spontaneously Scalarized Kerr Black Holes in Extended Scalar-Tensor-Gauss-Bonnet Gravity
}

\author{
Pedro V. P. Cunha, ${ }^{1,2}$ Carlos A. R. Herdeiro, ${ }^{2}$ and Eugen Radu ${ }^{1}$ \\ ${ }^{1}$ Departamento de Física da Universidade de Aveiro and CIDMA, Campus de Santiago, 3810-183 Aveiro, Portugal \\ ${ }^{2}$ Centro de Astrofísica e Gravitação-CENTRA, Departamento de Física, Instituto Superior Técnico-IST, \\ Universidade de Lisboa-UL, Avenida Rovisco Pais 1, 1049-001 Lisboa, Portugal
}

\section{(Received 26 April 2019; published 1 July 2019)}

\begin{abstract}
We construct asymptotically flat, spinning, regular on and outside an event horizon, scalarized black holes (SBHs) in extended scalar-tensor-Gauss-Bonnet models. They reduce to Kerr BHs when the scalar field vanishes. For an illustrative choice of nonminimal coupling, we scan the domain of existence. For each value of spin, SBHs exist in an interval between two critical masses, with the lowest one vanishing in the static limit. Non-uniqueness with Kerr BHs of equal global charges is observed; the SBHs are entropically favoured. This suggests that SBHs form dynamically from the spontaneous scalarization of Kerr BHs, which are prone to a scalar-triggered tachyonic instability, below the largest critical mass. Phenomenologically, the introduction of BH spin damps the maximal observable difference between comparable scalarized and vacuum BHs. In the static limit, (perturbatively stable) SBHs can store over 20\% of the spacetime energy outside the event horizon; in comparison with Schwarzschild BHs, their geodesic frequency at the ISCO can differ by a factor of 2.5 and deviations in the shadow areal radius may top $40 \%$. As the BH spin grows, low mass SBHs are excluded, and the maximal relative differences decrease, becoming of the order of a few percent for dimensionless spin $j \gtrsim 0.5$. This reveals a spin selection effect: non-GR effects are only significant for low spin. We discuss if and how the recently measured shadow size of the M87 supermassive BH constrains the length scale of the Gauss-Bonnet coupling.
\end{abstract}

DOI: 10.1103/PhysRevLett.123.011101

Introduction.-Strong gravity has entered the precision era. The breakthroughs in gravitational wave astrophysics $[1,2]$ and the unveiling of the first black hole (BH) shadow image [3-5] are probing the true nature of $\mathrm{BH}$ candidates. Thus, the hypothesis that astrophysical BHs when near equilibrium are well described by the Kerr metric [6] the Kerr hypothesis - can be tested to a new level of accuracy [7-9].

A primary concern in such testing is to assess degeneracy. That is, to what extent other viable BH models mimic the Kerr phenomenology. Three theoretical requirements for a viable BH model are that it should (i) arise in a consistent and well-motivated (effective field) theory of gravity, (ii) have a dynamical formation mechanism, and (iii) be (sufficiently) stable [10]. Thus, a pressing theoretical task is to investigate the phenomenology of models obeying these criteria.

One such family of models arises in the context of the BH spontaneous scalarization mechanism [11-13], akin to the scalarization of neutron stars occurring in scalar-tensor models [14]. By considering extended-scalar tensor GaussBonnet models (ESTGB), which include a Gauss-Bonnet (GB) term, vacuum General Relativity (GR) BHs-the Kerr family - may scalarize. On the one hand, this is a sound class of models with second order field equations, avoiding Ostrogradsky instabilities [15], and with high energy physics motivations [16]. On the other hand, the scalarization phenomenon yields a formation mechanism, and, in the only case studied hitherto - the static spherical case [17-25]—scalarized BHs (SBHs) stable against radial perturbations have been found [26,27]. In this Letter we report on ESTGB spinning SBHs, which may form from the spontaneous scalarization of Kerr BHs. We show that introducing (the astrophysically relevant) spin downsizes the phenomenological effects of scalarization. Using our results, we discuss how the M87 BH shadow measurement [5] may constrain the length scale of the ESTGB spontaneous scalarization models.

The model.-Nonminimal couplings often allow circumventing $\mathrm{BH}$ no-scalar hair theorems [28,29]. A dynamical scenario of $\mathrm{BH}$ spontaneous scalarization relies on three key ingredients. (a) One augments Einstein's GR with a new (real) scalar field degree of freedom, $\phi$, describing a spacetime varying coupling. The proposal that fundamental couplings vary in space and time is old, e.g., Refs. [30,31], and, in particular, motivated by different attempts at grand unification theories and quantum gravity. (b) One adds to GR a source of gravity, $\mathcal{I}$, which can trigger a repulsive gravitational effect for $\mathrm{BHs}$, and assumes some function $f(\phi)$ describes the coupling strength of $\mathcal{I}$. This class of models is described by the action: 


$$
S=\frac{1}{16 \pi} \int d^{4} x \sqrt{-g}\left[R-2 \partial_{\mu} \phi \partial^{\mu} \phi+f(\phi) \mathcal{I}\right],
$$

where $R$ is the Ricci scalar of the spacetime metric $g_{\mu \nu}$. (c) One chooses $f(\phi)$ so that both GR and non-GR (scalarized) exist in the model and the former may become unstable.

The choice of $\mathcal{I}$ in (ii) could be, e.g., the familiar electromagnetic Lagrangian $\mathcal{I}_{\mathrm{EM}}=-F_{\mu \nu} F^{\mu \nu}$ [32] or, the choice herein, the Gauss-Bonnet curvature squared term,

$$
\mathcal{I}_{\mathrm{GB}}=\lambda^{2}\left(R^{\mu \nu \alpha \beta} R_{\mu \nu \alpha \beta}-4 R_{\mu \nu} R^{\mu \nu}+R^{2}\right),
$$

where $\lambda$ is a constant length scale. Choosing $\mathcal{I}_{\mathrm{EM}}$, electrovacuum GR BHs become unstable below a certain $M / Q$, i.e., $\mathrm{BH}$ mass to charge ratio, determined by the choice of $f(\phi)$, and spontaneously scalarize. In this model, scalarization was established dynamically [32-34] and shown to be approximately conservative, for high $M / Q$. Choosing $\mathcal{I}_{\mathrm{GB}}$, vacuum GR BHs become unstable against scalarization below a certain $M / \lambda$ [11]. In both cases, it becomes dynamically favorable for BHs with sufficiently low mass compared to the length scale associated to the repulsive effect $(Q$ or $\lambda)$ to excite the scalar field, varying the coupling strength of the repulsive term.

Coupling function and instability threshold. - Appropriate couplings $f(\phi)$ obey $\left.f^{\prime}(\phi)\right|_{\phi=0} \equiv d f /\left.d \phi\right|_{\phi=0}=0$, so that the vacuum GR BHs are solutions of Eq. (1). Moreover, the latter are prone to a tachyonic instability triggered by a scalar field perturbation if $\mathcal{I} d^{2} f /\left.d \phi^{2}\right|_{\phi=0}>0$ [32]. A choice of appropriate coupling, which yields entropically favored, perturbatively stable, static, spherical SBHs in the ESTGB model, is as follows [11]:

$$
f(\phi)=\frac{1}{2 \beta}\left(1-e^{-\beta \phi^{2}}\right),
$$

where $\beta>0$. Following [11] we take $\beta=6$. For sufficiently high $\beta$, the properties of this particular choice are universal. For Schwarzschild $\mathrm{BHs}, \mathcal{I}_{\mathrm{GB}}>0$, and both above conditions are met for Eq. (3).

Schwarzschild $\mathrm{BHs}$ are unstable against scalarization when $[11,12] M / \lambda \lesssim 0.587$. This number is independent of the specific choice of $f(\phi)$, as long as it is compatible with scalarization. For a Kerr BH with given dimensionless spin $j \equiv J / M^{2}$, the corresponding threshold for stability is now a function of $J / \lambda^{2}$, forming an existence line in the $(M, J)$ plane. We will see in Fig. 1 that there exist some $J$, such that the threshold of scalarization is larger than 0.587 . Thus, spinning BHs can scalarize for (slightly) larger masses.

The domain of existence.-Stationary, axisymmetric solutions of Eq. (1) describing spinning, SBHs can be constructed using the metric ansatz $d s^{2}=-e^{2 F_{0}} N d t^{2}+$ $e^{2 F_{1}}\left(d r^{2} / N+r^{2} d \theta^{2}\right)+e^{2 F_{2}} r^{2} \sin ^{2} \theta(d \varphi-W d t)^{2}$, where $N \equiv 1-r_{H} / r$, and $F_{i}, W$, as well as the scalar field $\phi$,

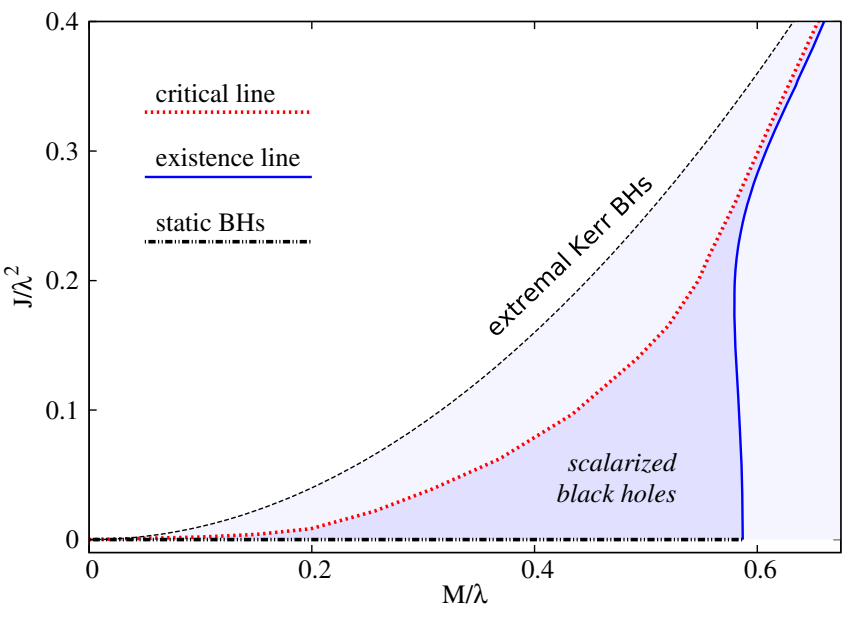

FIG. 1. $(M, J)$ domain of existence of spinning SBHs.

depend on $r, \theta$ only. Asymptotic flatness is guaranteed by imposing that $\lim _{r \rightarrow \infty} F_{i}=\lim _{r \rightarrow \infty} W=\lim _{r \rightarrow \infty} \phi=0$. Axial symmetry and regularity impose, on the symmetry axis, $\theta=0, \pi, \partial_{\theta} F_{i}=\partial_{\theta} W=\partial_{\theta} \phi=0$, and $F_{1}=F_{2}$, (no conical singularities). At the event horizon, located at a constant value of $r=r_{H}>0$, a new radial coordinate is convenient, $x \equiv \sqrt{r^{2}-r_{H}^{2}}$, leading to the horizon boundary conditions, at $r=r_{H}, \partial_{x} F_{i}=\partial_{x} \phi=0$ and $W=\Omega_{H}$, where the constant $\Omega_{H}>0$ is the horizon angular velocity.

The system of coupled PDEs obtained from Eq. (1) is solved using a Newton-Raphson relaxation method, the above boundary conditions and the same numerical strategy and solver as in, e.g., [35]. The ADM mass $M$ and total angular momentum $J$ of the BH solutions are read off from the asymptotic expansions, for large $r: g_{t t}=-e^{2 F_{0}} N+$ $e^{2 F_{2}} W^{2} r^{2} \sin ^{2} \theta \simeq-1+2 M / r, \quad g_{\varphi t}=-e^{2 F_{2}} W r^{2} \sin ^{2} \theta \simeq$ $-2 J \sin ^{2} \theta / r$. In Fig. 1, we exhibit the domain of existence of the spinning SBHs in the $(M, J)$ plane (shaded blue region), obtained by extrapolating to the continuum a discrete set of thousands of solutions. The domain of existence is bounded by the static BHs (black dashed line), which have $J=0$ and $M / \lambda<0.587$, the existence (blue solid) line corresponds to the Kerr BHs, which can support test field configurations of the scalar field (a zero mode of the instability [36]) and a set of critical solutions (red dotted line). The existence line is universal for any $f(\phi)$ allowing scalarization, cf. Ref. [34]. The critical and static sets are model dependent. The former reveals that below a certain $M / \lambda$, which depends on $J$, solutions cease to exist. This behavior is shared with the dilaton-GB model $[37,38]$. Physically, the repulsive GB term can prevent the existence of an event horizon below a certain $M / \lambda$, and this value increases with $J$, which adds another repulsive effect [39].

Physical properties.-Nonuniqueness between scalarized and Kerr BHs, for the same global quantities $(M, J)$, is manifest in Fig. 1. To get a measure of how much these two families of BHs differ, we show in Fig. 2 


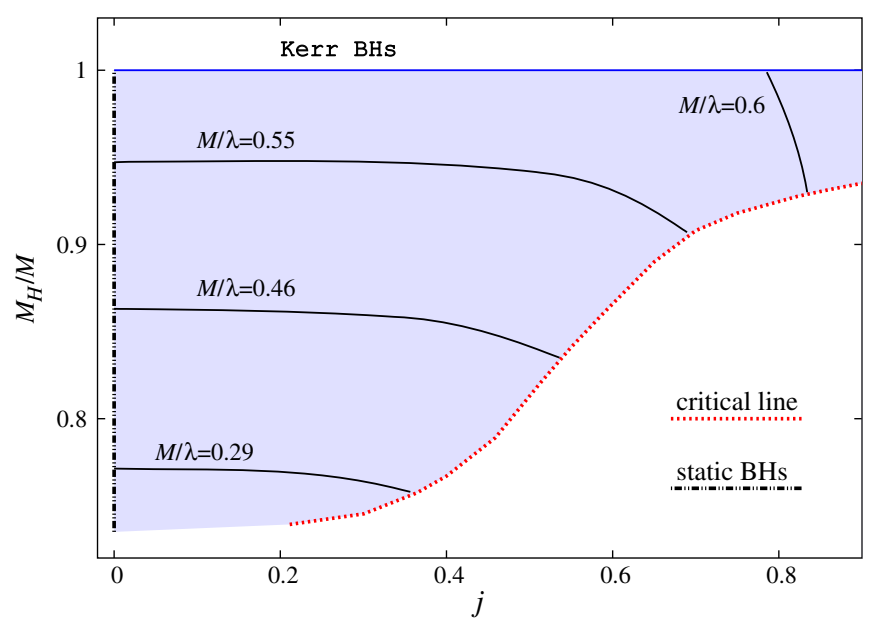

FIG. 2. Horizon mass to ADM mass ratio vs $j$. The color coding in Figs. $1-3$ is the same.

the ratio of the horizon mass, computed as the Komar integral [40] for the stationarity Killing vector field, $k=\partial_{t}$, to the ADM mass, as a function of the dimensionless spin. For static BHs $(j=0)$ over $20 \%$ of the spacetime energy can be stored outside the horizon. This occurs for solutions with the smallest values of $M / \lambda \lesssim 0.29$. For large spin, say $j \gtrsim 0.8$, solutions have $M / \lambda \gtrsim 0.6$ and the energy outside the horizon is less than $10 \%$. In the $j=0$ limit, the SBHs in this model are stable against radial perturbations for $M / \lambda \gtrsim$ 0.171 [26], which encompasses the whole static (black dashed) line in Fig. 2.

Let us assess the entropy of the spinning SBHs. In the ESTGB model, the $\mathrm{BH}$ entropy is not given by the Bekenstein-Hawking formula, $S_{\mathrm{BH}}=A_{H} / 4$, where the event horizon area $A_{H}$, is, in terms of our metric ansatz, $A_{H}=2 \pi r_{H}^{2} \int_{0}^{\pi} d \theta \sin \theta e^{F_{1}^{(2)}(\theta)+F_{2}^{(2)}(\theta)}$. The corrected entropy is obtained using Wald's approach [41],

$$
S=S_{\mathrm{BH}}+S_{\mathrm{sGB}}, \quad S_{\mathrm{sGB}} \equiv \frac{\lambda^{2}}{2} \int_{H} d^{2} x \sqrt{h} f(\phi) \mathrm{R}^{(2)},
$$

where $\mathrm{R}^{(2)}$ is the Ricci scalar of the metric $h_{i j}$, induced on the spatial sections of the event horizon, $H$. Defining the reduced (dimensionless) area and entropy,

$$
a_{H} \equiv \frac{A_{H}}{16 \pi M^{2}}, \quad s \equiv \frac{S}{4 \pi M^{2}},
$$

we plot these quantities in Fig. 3 for the spinning SBHs. For fixed $j$, the reduced area of the SBHs decreases from the existence to the critical line. In this sense, the SBHs are smaller than comparable Kerr BHs (with the same $M, J$ ). But, by virtue of the corrected entropy formula, the reduced entropy of the spinning SBHs, for fixed $j$, increases from the existence to the critical line, cf. Fig. 3 (inset).

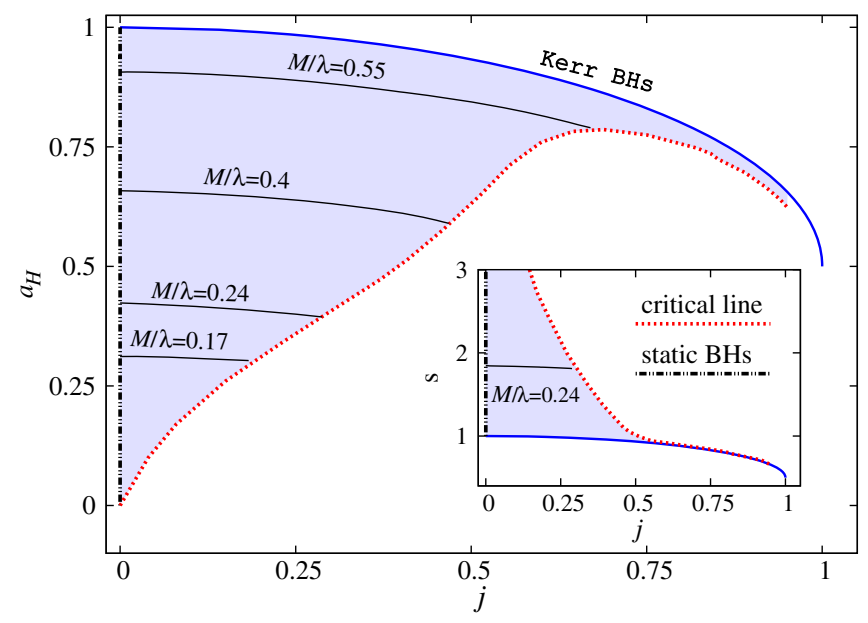

FIG. 3. Reduced area (main panel) and reduced entropy (inset) of the spinning SBHs vs $j$.

Thus, SBHs are entropically preferred over comparable, i.e., the same $(M, J)$, Kerr BHs.

The solutions obey a Smarr-type formula [42],

$$
M=2\left(T_{H} S+\Omega_{H} J\right)+M_{(\phi)},
$$

where $T_{H}=\left[e^{F_{0}^{(2)}(\theta)-F_{1}^{(2)}(\theta)}\right] /\left(4 \pi r_{H}\right)$ is the Hawking temperature and $M_{(\phi)}$ is a bulk (outside the horizon) integral along a spacelike hypersurface $\Sigma$, accounting for the scalar field $M_{(\phi)}=(1 / 2 \pi) \int_{\Sigma} d^{3} x \sqrt{-g}\left(f(\phi) / f^{\prime}(\phi)\right) \square \phi$. This formula is used for numerical accuracy tests [43]. Another test is provided by the first law of BH thermodynamics, $d M=T_{H} d S+\Omega_{H} d J$, where no explicit scalar field term appears, even though the solutions possess a scalar "charge" $Q_{s}$, which is read off from the far-field asymptotics as $\phi \simeq-Q_{s} / r$.

BH shadows. - The shadow is an optical image of a $\mathrm{BH}$ due to background or surrounding light sources [44-48]. Recently the first image of a $\mathrm{BH}$ shadow was released [3-5]. A BH shadow is a feature of strong gravitational lensing, and it is determined by the fundamental photon orbits-bound states of light around the BH [49]. These include, in particular, the equatorial light rings (LRs). Measuring the LRs gives the boundary of the shadow. The shadows in two distinct (stationary and axisymmetric) $\mathrm{BH}$ spacetimes are comparable if the BHs are comparable and the observers are identical, say, both on the equatorial plane and at the same perimetral distance $\sqrt{g_{\varphi \varphi}}$ from each BH [50]. In Fig. 4 the shadow and lensing of two SBHs, and their comparable vacuum counterparts are shown, obtained using ray tracing. The $j=0 \mathrm{SBH}$ is the smallest stable one [26], in order to maximize the relative difference with its vacuum counterpart. The shadow of the former has roughly half the size, whereas the Einstein ring (the lensing of the image point behind the $\mathrm{BH}$ ) is similar (top panels, Fig. 4). For the spinning SBH and Kerr BH (both with the 


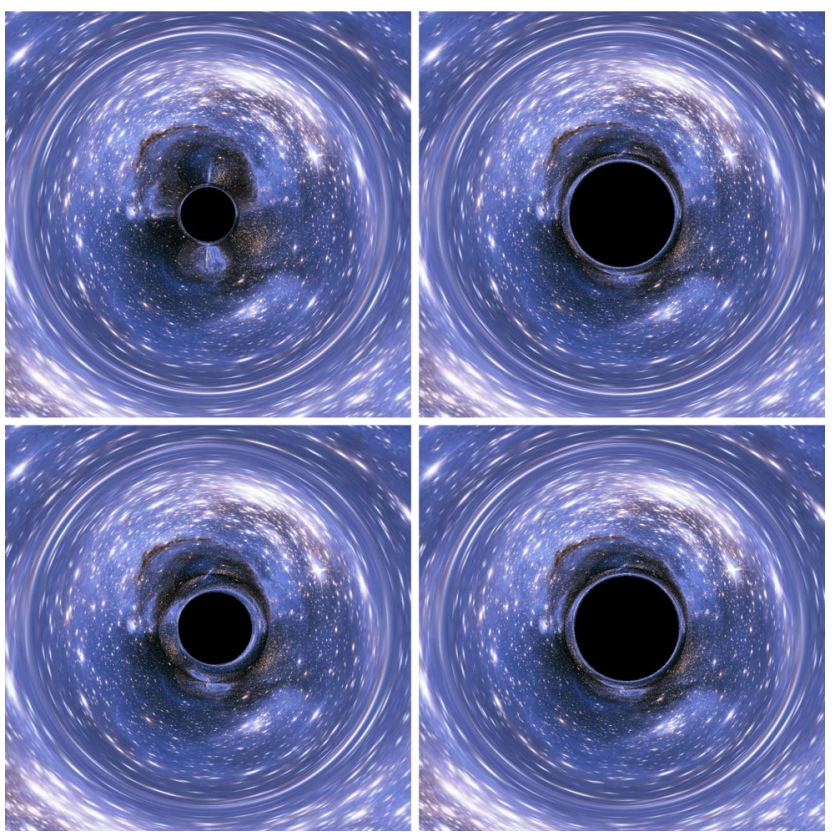

FIG. 4. Shadows and lensing, under comparable observation conditions. Top, left to right: (i) static SBH with $M / \lambda \sim 0.172$, (ii) comparable Schwarzschild BH. Bottom, left to right: (iii) spinning SBH with $M / \lambda \sim 0.237$ ( $j=0.24$ ), (iv) comparable Kerr $\mathrm{BH}$. The background image can be found in Ref. [51].

spin value, $j=0.24$ ) the differences are still obvious, but smaller (lower panels, Fig. 4). To make the comparison quantitative, we introduce the areal radius $\bar{r} \equiv \sqrt{A / \pi}$, for a shadow image with area $A ; \bar{r}$ is well defined even for noncircular shadows. This measure is used in Fig. 5 to obtain the relative deviation in the shadow size between SBHs and Kerr BHs. One observes the deviations increase monotonically as $M / \lambda$ decreases. For $j=0$, deviations can be larger than $80 \%$, or, restricting to the stable $\mathrm{BHs}$ $(M / \lambda \gtrsim 0.171)$, larger than $40 \%$. This is consistent with SBHs being smaller, cf. Fig. 3. Turning on $j$ introduces a critical lower mass, cf. Fig. 1, and the larger deviations (at the smallest $M / \lambda$ ) are absent. For $j=0.24$, deviations still reach $\sim 30 \%$, but for $j=0.48$, deviations are only of a few percent, resembling the Einstein-dilaton-GB model [46]. The $j=0.24 \mathrm{SBH}$ shown in Fig. 4 maximizes the difference with Kerr for this spin value. For $j \gtrsim 0.5$ the shadows of SBHs differ from Kerr $\lesssim 2 \%$ and lensing images of this sort would look identical to the comparable Kerr.

The M87 shadow.-The M87 supermassive $\mathrm{BH}$ imaging [3] gave a $\mathrm{BH}$ angular scale of $\theta_{g}=3.8 \pm 0.4 \mu$ as. We thus consider a $10 \%$ error in the measured shadow size [52]. A prior measurement of the $\mathrm{BH}$ mass using stellar dynamics [53], but with updated distance [3], gave $M_{\mathrm{M} 87}=$ $6.2_{-0.6}^{+1.1} \times 10^{9} M_{\odot}$. Taking this as the true mass, a putative SBH is only consistent with the data (which is consistent with a Kerr BH) if the relative shadow deviation is $\lesssim 10 \%$. The corresponding line separating excluded from viable SBHs is shown in Fig. 5. Its intersection with the line of

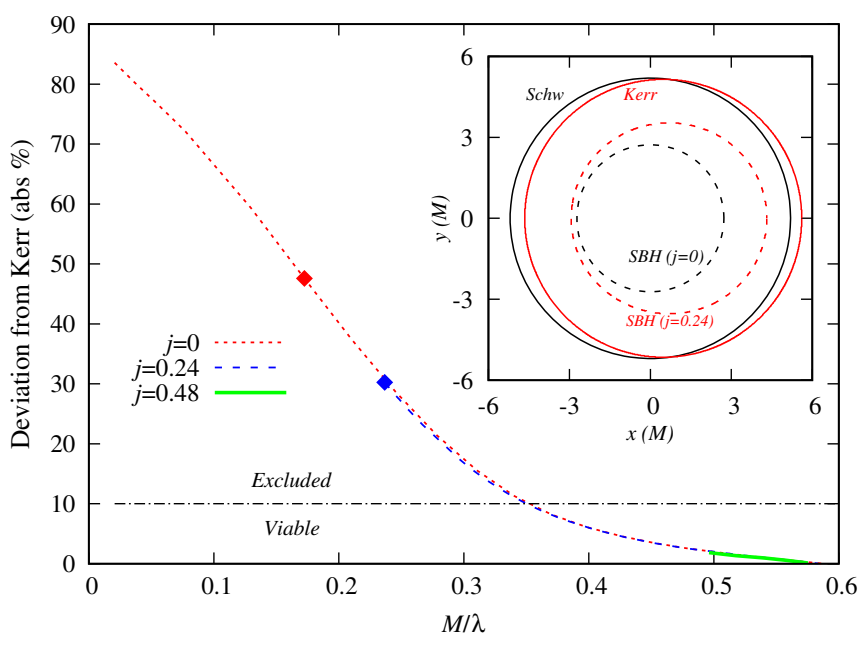

FIG. 5. Shadow size relative deviations between SBHs and Kerr BHs. (Right inset) contours of the shadows in Fig. 4 for comparison; the two SBHs are highlighted as diamonds in the main panel.

solutions is not very sensitive to $j: M / \lambda=0.35$ (0.353) for $j=0(j=0.24)$. Such low spin values are compatible with some estimates of the M87 BH spin, say, $j \sim 0.1$ in Ref. [54]. Imposing $M_{\mathrm{M} 87} / \lambda \gtrsim 0.353$, yields the (weak) constraint $\lambda \lesssim 1.8 \times 10^{10} M_{\odot}$. For larger $\lambda$, a BH with $M=$ $M_{\mathrm{M} 87}$ would scalarize, and, assuming this process to be approximately conservative [55], the SBH shadow would be too small. Even though the mass estimate in Ref. [53] assumes GR, the GB term fall off as $\sim 1 / r^{6}$ implies GR is a good approximation of a few gravitational radii in our model and thus in the study of stellar dynamics. On the other hand, if the M87 BH spin is large, as in other estimates, say $j \sim 0.9$ in Ref. [56], its shadow measurement [3] per se is compatible with a $\mathrm{SBH}$, as differences are below the $10 \%$ error bar.

Frequency at the ISCO.- The geodesic frequency at the innermost stable circular orbit (ISCO) of the SBHs, $\Omega$, is of relevance for x-ray astronomy, since the inner edge of accretion disks is often modeled at the ISCO (e.g., ThorneNovikov disk model [57]). Then this frequency dictates the cutoff on the frequency of synchroton radiation, which is used in the continuum fitting of the x-ray spectrum of accreting BH systems [58]. The ISCO frequency computation follows, e.g., Ref. [35]. Figure 6 shows the relative frequencies at the ISCO for SBHs and Kerr BHs. For $j=0$, the ratio $\Omega^{(s)} / \Omega^{(\mathrm{GR})}$ reaches a maximum $\sim 2.5$, attained for stable BHs. The transition to unstable BHs is marked with a square $(M / \lambda \lesssim 0.171)$. In the spinning case, for $j=0.24$, deviations are larger for prograde orbits [denoted with $(+)$ ] with a maximum ratio $\sim 2.3$. For $j=0.48$ (inset) deviation are $\lesssim 10 \%$, and even less for higher $j$. For $j=0, r_{\text {ISCO }}$ and $r_{L R}$ have a different sensitivity to $M / \lambda$. Varying $M / \lambda$ from $0.587 \rightarrow 0.3$, the former approximately halves, whereas the latter becomes only $\sim 2 / 3$ of its initial value. This explains 


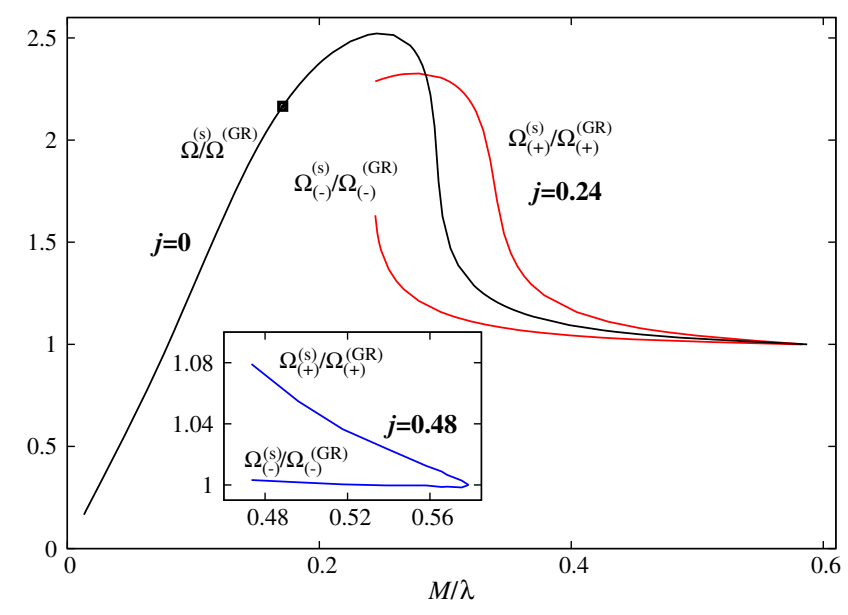

FIG. 6. Relative frequencies at the ISCO for scalarized and GR BHs.

the larger deviation from Schwarzschild in the ISCO behavior as compared to shadows.

Universality and a spin selection effect. - Other coupling functions $f(\phi)$ compatible with spontaneous scalarization, e.g., Ref. [12], imply a different range for $M / \lambda$ even in the static $(j=0)$ case. As a naïve expectation, the impact of $j \neq 0$ should generically follow the trend herein: increase the minimal value of $M / \lambda$ and simultaneously decrease the maximal relative deviations from Kerr, as in Ref. [46]. This contrasts with the effect of spin in some models of scalarized stars [59].

Finally, we observe this model illustrates a spin selection effect: BHs with moderate to large spins will be either Kerr $\mathrm{BHs}$ or SBHs which are indistinguishable from Kerr BHs in current observations. Only low spin BHs could clearly unveil the scalarization phenomenon. In this regard, one may recall that typical $\mathrm{BHs}$ observed by both gravitational wave observations (as the final $\mathrm{BH}$ ) and $\mathrm{x}$-ray spectroscopy methods are estimated to have a fairly large spin: cf. Table III in Ref. [2] and Table 8.1 in Ref. [58].

P. C. is supported by Grant No. PD/BD/114071/2015 under the FCT-IDPASC Portugal Ph.D. program. This work is supported by the Fundação para a Ciência e a Tecnologia (FCT) Project No. UID/MAT/04106/2019 (CIDMA), by CENTRA (FCT) strategic Project No. UID/FIS/00099/2013, by national funds (OE), through FCT, I. P., in the scope of the framework contract foreseen in the numbers 4, 5, and 6 of the article 23, of the DecreeLaw 57/2016, of August 29, changed by Law 57/2017, of July 19. The project PTDC/FIS-OUT/28407/2017 is funded by FCT. This work has further been supported by the European Union's Horizon 2020 research and innovation (RISE) programmes H2020-MSCA-RISE2015 Grant No. StronGrHEP-690904 and H2020MSCA-RISE-2017 Grant No. FunFiCO-777740. The authors would like to acknowledge networking support by the COST Action CA16104.
[1] B. P. Abbott et al. (Virgo and LIGO Scientific Collaborations), Phys. Rev. Lett. 116, 061102 (2016).

[2] B. P. Abbott et al. (LIGO Scientific and Virgo Collaborations), arXiv:1811.12907.

[3] K. Akiyama et al. (Event Horizon Telescope), Astrophys. J. 875, L4 (2019).

[4] K. Akiyama et al. (Event Horizon Telescope), Astrophys. J. 875, L5 (2019).

[5] K. Akiyama et al. (Event Horizon Telescope), Astrophys. J. 875, L6 (2019).

[6] R. P. Kerr, Phys. Rev. Lett. 11, 237 (1963).

[7] E. Berti, E. Barausse, V. Cardoso, L. Gualtieri, P. Pani et al., Classical Quantum Gravity 32, 243001 (2015).

[8] V. Cardoso and L. Gualtieri, Classical Quantum Gravity 33, 174001 (2016).

[9] L. Barack et al., arXiv:1806.05195.

[10] J. C. Degollado, C. A. R. Herdeiro, and E. Radu, Phys. Lett. B 781, 651 (2018).

[11] D. D. Doneva and S. S. Yazadjiev, Phys. Rev. Lett. 120, 131103 (2018).

[12] H. O. Silva, J. Sakstein, L. Gualtieri, T. P. Sotiriou, and E. Berti, Phys. Rev. Lett. 120, 131104 (2018).

[13] G. Antoniou, A. Bakopoulos, and P. Kanti, Phys. Rev. Lett. 120, 131102 (2018).

[14] T. Damour and G. Esposito-Farese, Phys. Rev. Lett. 70, 2220 (1993).

[15] M. Ostrogradsky, Mem. Acad. St. Petersbourg 6, 385 (1850).

[16] B. Zwiebach, Phys. Lett. 156B, 315 (1985).

[17] G. Antoniou, A. Bakopoulos, and P. Kanti, Phys. Rev. D 97 , 084037 (2018).

[18] M. Minamitsuji and T. Ikeda, Phys. Rev. D 99, 044017 (2019).

[19] A. Bakopoulos, G. Antoniou, and P. Kanti, Phys. Rev. D 99, 064003 (2019).

[20] H. Motohashi and S. Mukohyama, Phys. Rev. D 99, 044030 (2019).

[21] Y. Brihaye and L. Ducobu, arXiv:1812.07438.

[22] C. F. B. Macedo, J. Sakstein, E. Berti, L. Gualtieri, H. O. Silva, and T. P. Sotiriou, Phys. Rev. D 99, 104041 (2019).

[23] D. D. Doneva, K. V. Staykov, and S. S. Yazadjiev, Phys. Rev. D 99, 104045 (2019).

[24] Y. S. Myung and D.-C. Zou, arXiv:1903.08312.

[25] Y. Brihaye and B. Hartmann, Phys. Lett. B 792, 244 (2019).

[26] J. L. Blázquez-Salcedo, D. D. Doneva, J. Kunz, and S. S. Yazadjiev, Phys. Rev. D 98, 084011 (2018).

[27] H. O. Silva, C. F. B. Macedo, T. P. Sotiriou, L. Gualtieri, J. Sakstein, and E. Berti, Phys. Rev. D 99, 064011 (2019).

[28] T. P. Sotiriou and V. Faraoni, Phys. Rev. Lett. 108, 081103 (2012).

[29] C. A. R. Herdeiro and E. Radu, Int. J. Mod. Phys. D 24, 1542014 (2015).

[30] P. A. M. Dirac, Proc. R. Soc. A 165, 199 (1938).

[31] C. Brans and R. H. Dicke, Phys. Rev. 124, 925 (1961).

[32] C. A. R. Herdeiro, E. Radu, N. Sanchis-Gual, and J. A. Font, Phys. Rev. Lett. 121, 101102 (2018).

[33] Y. S. Myung and D.-C. Zou, Phys. Lett. B 790, 400 (2019).

[34] P. G. S. Fernandes, C. A. R. Herdeiro, A. M. Pombo, E. Radu, and N. Sanchis-Gual, arXiv:1902.05079.

[35] C. Herdeiro and E. Radu, Classical Quantum Gravity 32, 144001 (2015). 
[36] The zero mode is a perturbation mode with a vanishing imaginary part of the frequency.

[37] P. Kanti, N. E. Mavromatos, J. Rizos, K. Tamvakis, and E. Winstanley, Phys. Rev. D 54, 5049 (1996).

[38] B. Kleihaus, J. Kunz, S. Mojica, and E. Radu, Phys. Rev. D 93, 044047 (2016).

[39] Technically, this limit can be understood from the perturbative expansion of the solutions near the horizon [38] (see also Refs. [11-13] for the static limit), wherein the radicand of a square root becomes negative as some limiting solutions are approached, while the solver diverges. The collection of these limiting configurations forms the critical line.

[40] A. Komar, Phys. Rev. 113, 934 (1959).

[41] R. M. Wald, Phys. Rev. D 48, R3427 (1993).

[42] L. Smarr, Phys. Rev. Lett. 30, 71 (1973); 30, 521(E) (1973).

[43] Based on the accuracy with which the Smarr law and the first law of thermodynamics are obeyed, the numerical error for the $\mathrm{BH}$ solutions in this Letter is estimated to be typically $<10^{-3}$. This error agrees with the estimate provided by the solver.

[44] V. Perlick, Living Rev. Relativity 7, 9 (2004).

[45] P. V. P. Cunha and C. A. R. Herdeiro, Gen. Relativ. Gravit. 50, 42 (2018).

[46] P. V. P. Cunha, C. A. R. Herdeiro, B. Kleihaus, J. Kunz, and E. Radu, Phys. Lett. B 768, 373 (2017).

[47] H. Falcke, F. Melia, and E. Agol, Astrophys. J. 528, L13 (2000).

[48] T. Johannsen, C. Wang, A. E. Broderick, S. S. Doeleman, V. L. Fish, A. Loeb, and D. Psaltis, Phys. Rev. Lett. 117, 091101 (2016).
[49] P. V. P. Cunha, C. A. R. Herdeiro, and E. Radu, Phys. Rev. D 96, 024039 (2017).

[50] P. V. P. Cunha, C. A. R. Herdeiro, E. Radu, and H. F. Runarsson, Int. J. Mod. Phys. D 25, 1641021 (2016).

[51] NASA/Hubble, Background image used for lensing, http:// hubblesite.org/image/3905/printshop.

[52] The corresponding Schwarzschild shadow diameter is $6 \sqrt{3}+\theta_{g} \simeq 39 \mu$ as. For a Kerr $\mathrm{BH}$ this value varies, increasing $j$, by $\lesssim 2 \%$, due to the spin inclination angle $\sim 17^{\circ}$. There is an offset between such light ring diameter and the measured emission ring of $42 \pm 3 \mu$ as; $\theta_{g}$ is inferred from the latter via GRMHD models [5], which predict a peak emission slightly outside the light ring.

[53] K. Gebhardt, J. Adams, D. Richstone, T. R. Lauer, S. M. Faber, K. Gultekin, J. Murphy, and S. Tremaine, Astrophys. J. 729, 119 (2011).

[54] E. E. Nokhrina, L. I. Gurvits, V. S. Beskin, M. Nakamura, K. Asada, and K. Hada, arXiv:1904.05665.

[55] This is supported by the qualitatively similar dynamical processes studied in the cousin model [32].

[56] F. Tamburini, B. Thidé, and M. Della Valle, arXiv:1904 .07923

[57] I. D. Novikov and K.S. Thorne, in Black Holes (Les Astres Occlus) (Gordon and Breach, New York, 1973), pp. 343-450.

[58] C. Bambi, Black Holes: A Laboratory for Testing Strong Gravity (Springer Nature, Singapore, 2017).

[59] D. D. Doneva, S. S. Yazadjiev, N. Stergioulas, and K. D. Kokkotas, Phys. Rev. D 98, 104039 (2018). 